\title{
Forward growth of ferroelectric domains with charged domain walls. Local switching on non-polar cuts
}

\author{
Cite as: J. Appl. Phys. 129, 044103 (2021); doi: 10.1063/5.0037680 \\ Submitted: 15 November 2020 - Accepted: 17 January 2021 . \\ Published Online: 28 January 2021
}

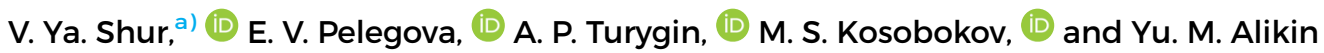

\author{
AFFILIATIONS \\ School of Natural Sciences and Mathematics, Ural Federal University, 620000 Ekaterinburg, Russia
}

Note: This paper is part of the Special Topic on Domains and Domain Walls in Ferroic Materials.

a) Author to whom correspondence should be addressed: vladimir.shur@urfu.ru

\begin{abstract}
Forward domain growth representing one of the main stages of domain switching is studied for isolated domains and domain arrays appearing as a result of tip-induced switching on the non-polar cuts of lithium niobate crystals. Formation of the wedge-like domains with a high aspect ratio and charged domain walls is observed. The domain growth in the area with a negligible external field is considered in terms of the kinetic approach based on analogy with crystal growth. The domain wall motion by step generation and propagation of the charged kinks is discussed. It is proposed that the switching field contains the inputs of the external field produced by a biased scanning probe microscope tip, the depolarization field produced by charged kinks, and the screening fields. According to the simulation results of the field distribution, the forward growth is caused by the step generation near the tip and the kink propagation induced by the depolarization field produced by the kinks. Scanning with the biased tip creates self-assembled domain arrays with several modes of domain length alteration: doubling, quadrupling, and chaotic. The statistical characterization of the arrays proves their high ordering. The array is formed under the influence of the depolarization field produced by three neighboring domains. The proposed mechanism can be applied for forward domain growth during switching on the polar cuts as well. In this case, the steps on the domain wall are generated on the polar surface, whereas the domain elongates by kink motion in the field produced by the charged kinks.
\end{abstract}

Published under license by AIP Publishing. https://doi.org/10.1063/5.0037680

\section{INTRODUCTION}

Ferroelectric materials have demonstrated a rich set of useful properties, including piezoelectricity, high nonlinear optical activity, pyroelectricity, and many others. The characteristics important for application depend on the domain structure. ${ }^{1}$ It has been shown that domain engineering and domain wall engineering allow improving the device performance of the commercially available ferroelectrics by creation of the stable tailored domain patterns, which introduce the spatial modulation of the main properties. ${ }^{2-4}$ The domain engineering is focused first on the fabrication of electro-optical and nonlinear optical devices, such as laser frequency converters based on periodically poled lithium niobate $\left(\mathrm{LiNbO}_{3}, \mathrm{LN}\right) .^{5-8}$ Creation of the domain patterns with desired parameters requires a deep understanding of the physical basis of the domain structure evolution during polarization reversal, ${ }^{8}$ which needs a development of the domain imaging methods.
The first imaging of the static domain structure in ferroelectrics was realized by optical microscopy in Rochelle salt ${ }^{9}$ and barium titanate $\left(\mathrm{BaTiO}_{3}, \mathrm{BT}\right)^{10}$ crystals. In situ domain imaging in BT by optical microscopy during the field induced polarization reversal by $\mathrm{Merz}^{11}$ and Little $^{12}$ allowed revealing the forward domain growth. Afterward, the domain structure was studied extensively in various ferroelectrics by modern high-resolution methods, such as scanning electron microscopy $(\mathrm{SEM})^{13}$ and piezoresponse force microscopy (PFM). ${ }^{14,15}$ However, the common research of the domain kinetics in the polar-cut samples (cut perpendicular to the polar axis) does not allow obtaining the direct information about domain growth in the crystal bulk. Thus, the fast domain growth in the polar direction (forward growth) is still insufficiently studied due to spatial resolution limitations of the optical microscopy and inapplicability of high-resolution methods. The transmission electron microscopy (TEM) has been applied 
recently for real-time atomic-scale observations of the forward domain growth in PZT thin films. ${ }^{16}$

The domain structure evolution during polarization reversal from the single-domain state consists of five main stages: (1) nucleation of new domains, (2) forward domain growth, (3) sideways domain growth, (4) domain merging, and (5) spontaneous backswitching after external field switch-off (Fig. 1). ${ }^{17}$ The forward growth of the domains with charged walls plays an essential role in two main stages. The domains appearing on the polar surfaces grow rapidly in a polar direction during the forward growth stage [Fig. 1(b)], and the elongation of non-through residual domains is revealed at the backswitching stage [Fig. 1(e)].

In situ imaging of the forward growth in polar-cut samples is hampered by existence of a number of independently growing domains situated in the crystal bulk and their very fast growth through the sample. The used imaging of the static domain structure at cross sections is ineffective for detailed research because the images of needle-like domains are distorted by any deviation of the cross section from the polar direction. ${ }^{18}$ Moreover, a selective chemical etching used for domain revealing can change the domain shape significantly. ${ }^{19}$

The recent observation of the in-plane domain growth in the non-polar-cut samples (cut parallel to the polar axis) of uniaxial ferroelectrics by means of PFM imaging during local switching by a biased conductive tip of a scanning probe microscope (SPM) has allowed direct study of the forward growth with nanoscale spatial resolution. ${ }^{20}$ The advantages of the method include the possibility to study the nucleation and growth of the single domain on the crystal surface with the determined position of the nucleation site. The switching and high-resolution imaging by PFM are realized by the same conductive tip. Moreover, the SEM imaging with nanoscale resolution can be used.

The local polarization reversal on the non-polar cuts was studied in LN by a local application of the electric field using a metallic needle, ${ }^{21}$ an electron beam, ${ }^{22,23}$ and a conductive SPM tip. $^{20,24,25}$ It was shown that on the non-polar cuts, the backswitching after the termination of the external field led to either change in the switched domain shape or even formation of the domain oriented in the opposite direction. ${ }^{24}$ A key role of the local charge injection $^{25-30}$ and screening of the applied electric field ${ }^{31-34}$ was also demonstrated. The effects of domain interaction and selforganization during local switching by a biased SPM tip on the non-polar cut of MgOLN were studied by us earlier and attributed to the influence of the electric field localized at the charged domain walls (CDWs) of the wedge-like domains. ${ }^{25,28}$

The LN is uniaxial crystal with $\boldsymbol{C}_{\mathbf{3 v}}(\boldsymbol{R} \mathbf{3} \boldsymbol{c})$ symmetry in the ferroelectric phase and the domain structure with $180^{\circ}$ domain walls only. The pronounced electro-optical effect allows applying the direct optical methods for in situ observation of the domain kinetics. ${ }^{35}$ Moreover, LN has recently become the recognized leader in the study of the domain structure stimulated by practical reasons. It is possible to claim that now $\mathrm{LN}$ can be used also as a model crystal for studying the domain kinetics. CLN crystals doped by $\mathrm{MgO}(\mathrm{MgOLN})$ in order to increase essentially the optical damage threshold are widely produced. MgOLN crystals are crucial for periodically poled elements for high power light conversion. The crystals of the LN family are the first and still the most widely exploited periodically poled ferroelectrics and the most desirable material for micro- and nanodomain engineering.

The existing theoretical approach to the domain forward growth is based on the ferroelectric domain breakdown model proposed by Molotskii et al. ${ }^{36,37}$ They explain the experimentally observed domain growth through the polar-cut thick crystal during local switching by a biased conductive SPM tip. They have claimed that "the domain breakdown is considered an extreme manifestation of Coulomb instability, ... which leads to strong pulling of the domain apex into a region where the tip-induced electric field is negligibly small." 37 According to their model, "the main driving force for ferroelectric domain breakdown is ... rather internal force generated owing to the minimization of depolarization field energy when the domain elongates." 37

Thus, the main idea of the ferroelectric domain breakdown is based on the statement that existence of the CDW is forbidden due to increase in the depolarization energy. However, it is known
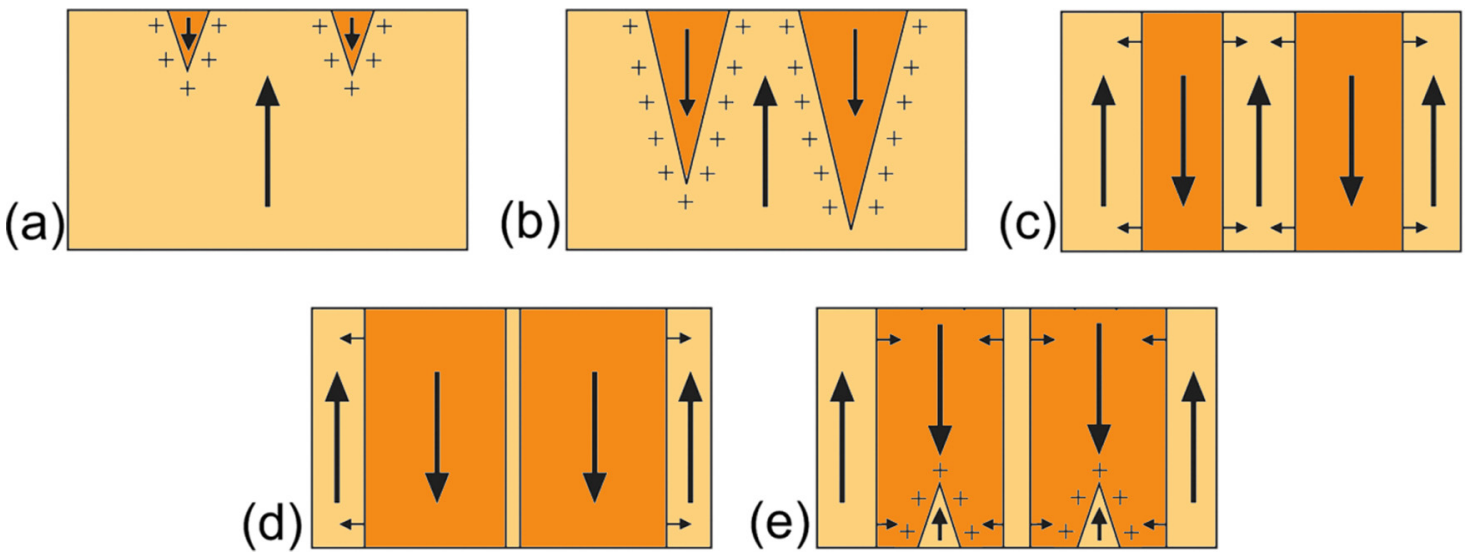

(e)

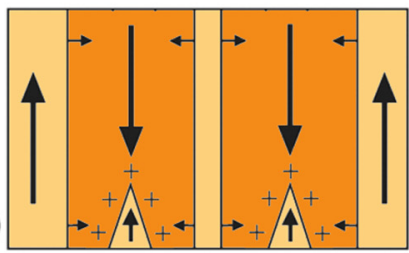

FIG. 1. The main stages of domain structure evolution during polarization reversal: (a) nucleation of new domains, (b) forward domain growth, (c) sideways domain growth, (d) coalescence of residual domains, and (e) spontaneous backswitching. 
nowadays that this postulate is wrong and the metastable domain structures with CDW are extensively studied in various ferroelectrics both experimentally and theoretically. ${ }^{38-41}$

The formation of the nanodomain with in-plane polarization in non-polar-cut samples of uniaxial ferroelectrics LN and lithium tantalate by SPM was theoretically considered by Pertsev andKholkin. ${ }^{42}$ The calculated equilibrium sizes and a wedge-like shape of the subsurface nanodomains oriented along the polar axis were attributed to a spatial distribution of the polar component of the electric field. Later, the unexpectedly long domains compared to the theoretical estimations were obtained experimentally. ${ }^{20}$

Here, we studied experimentally the forward domain tip-induced polarization switching on non-polar cuts of CLN and MgOLN single crystals. Formation of the self-assembled domain arrays as a result of scanning with a biased SPM tip was also investigated.

\section{EXPERIMENTAL}

We studied the tip-induced polarization switching on the $\mathrm{X}$ - and Y-non-polar cuts in single crystals of CLN and MgOLN. The $400 \mu \mathrm{m}$ thick CLN plates were used for studying the selfassembling and $400 \mu \mathrm{m}$ and $1 \mathrm{~mm}$ thick MgOLN plates for point switching. All wafers were produced by Yamaju Ceramics, Japan. The surface roughness of all samples was about $1 \mathrm{~nm}$.

Local polarization reversal was realized using NI6251 multifunction Data Acquisition board (National Instruments, USA) and a high-voltage amplifier Trek-677B (TREK, Inc., USA). PFM was used for domain visualization on the sample surface. The domain structure was created and visualized using Probe NanoLaboratory NTEGRA Aura (NT-MDT, Spectrum Instruments, Russia). Commercial NSC18 probes with titanium-platinum conductive coating (MikroMasch, Estonia) were used with a curvature radius of $35 \mathrm{~nm}$, a resonance frequency of $70 \mathrm{kHz}$, and a spring constant of $3.5 \mathrm{~N} / \mathrm{m}$. PFM measurements were carried out using $3 \mathrm{~V} \mathrm{AC}$ voltage with frequency far from the contact resonance of the tip.

Two types of domain structures were studied: isolated domains and domain arrays. The isolated domains were created by point switching with the single rectangular pulses. The pulse amplitude $U_{p}$ ranged from $40 \mathrm{~V}$ to $200 \mathrm{~V}$ and the pulse duration $t_{p}$ ranged from $10 \mathrm{~ms}$ to $10 \mathrm{~s}$. The withdraw switching mode was realized. ${ }^{24}$ The SPM tip was withdrawn from the surface with applied bias before termination of the switching pulse. The domain arrays were produced by scanning with the biased SPM tip moving with a fixed velocity of $1 \mu \mathrm{m} / \mathrm{s}$ and an applied voltage ranging from 100 to $250 \mathrm{~V}$. All experiments were performed at room temperature in dry nitrogen flow with relative humidity $(\mathrm{RH})$ below $4 \%$.

\section{RESULTS AND DISCUSSION}

\section{A. Single domain}

During point switching, the wedge-like domains appear near the tip in the area with the direction of the field polar component $E_{z}$ being opposite to spontaneous polarization and grow in the polar Z-direction [Figs. 2(a) and 2(b)]. The typical domain shape is presented in Fig. 2(c).

The domain length and width increase linearly with the switching pulse amplitude [Figs. 3(a) and 3(c)] and logarithmically (a)
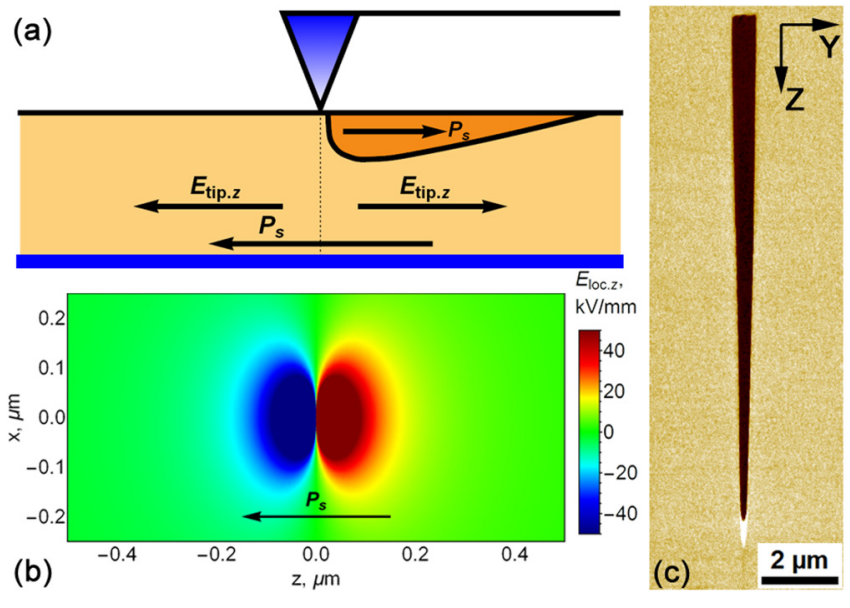

FIG. 2. (a) A scheme of the tip-induced polarization reversal on non-polar cut of a uniaxial ferroelectric. (b) A spatial distribution of the polar component of the electric field produced by a biased SPM tip. A blue line at the bottom represents the solid bottom electrode. (c) PFM image of a typical domain formed on an $\mathrm{X}$-cut of $400-\mu \mathrm{m}$-thick MgOLN as a result of tip-induced switching by a single rectangular pulse with amplitude $U_{p}=200 \mathrm{~V}$ and duration $t_{p}=0.5 \mathrm{~s}$.

with pulse duration [Figs. 3(b) and 3(d)]. Similar dependences of the domain radius on the pulse amplitude and duration are typical for local domain switching on the polar cuts. ${ }^{43,44}$

It is necessary to point out that the maximum domain length on Y-cut reached $30 \mu \mathrm{m}$, thus drastically exceeding the theoretical prediction $^{42}$ [Fig. 3(e)]. However, the polar component of the electric field produced by the SPM tip localized in a small area near the tip is negligibly small at such long distances. Moreover, the formation of the domains with charged walls contradicts the basic idea of the theoretical model of Molotskii et al. ${ }^{36,37}$ Therefore, we used the kinetic approach to explain the obtained experimental results. ${ }^{17,45,46}$

The mechanism of tip-induced polarization reversal on the nonpolar cut of a uniaxial ferroelectric is fundamentally different from the one on the polar cut. It is caused by the spatial distribution of the electric field lateral component induced by the SPM tip [Fig. 2(b)], while the direction of spontaneous polarization in the initial singledomain state is uniform along the whole sample. The spatial field distribution leads to an unusual domain growth [Fig. 3(f)].

According to the kinetic approach, the domain structure evolution representing an example of solid-solid first order phase transformation is determined by nucleation processes. ${ }^{47,48}$ It was pointed out earlier that the mechanism of crystal growth could be applied "not only for surfaces between a solid and a fluid, but also for interfaces in a crystalline medium when the same lattice extends to the both phases as in ... ferroelectric domain walls." ${ }^{49}$ The domains divided by domain walls are similar to the volumes of different phases divided by interfaces. Thus, the growth of the ferroelectric domain can be considered in analogy with crystal growth from the melt. The evolution of the domain structure in the electric field is caused by generation of various nuclei with preferred orientation of spontaneous polarization (Fig. 3). The nucleation rates are proportional to an excess of the local electric field over the threshold values. 

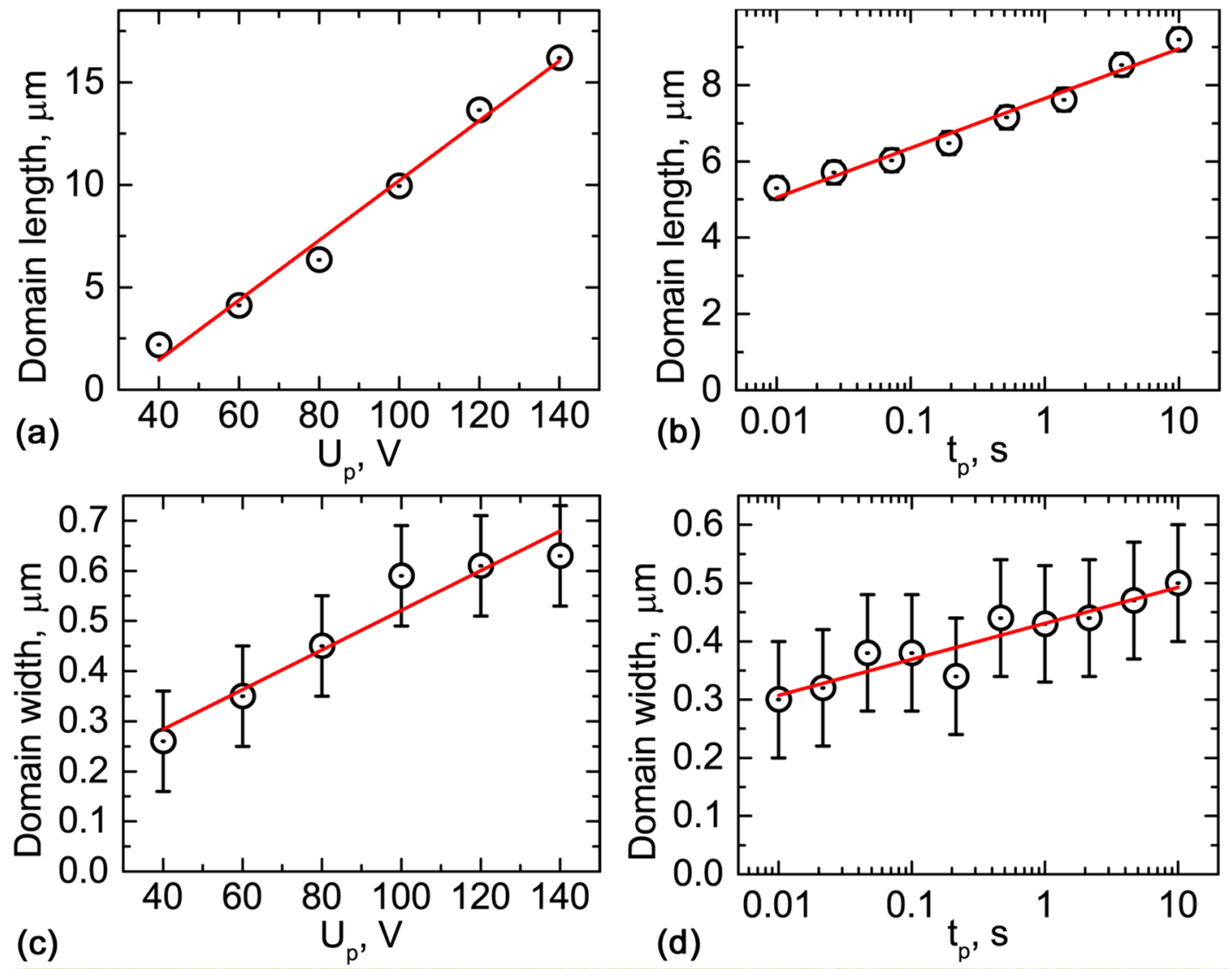

(e)
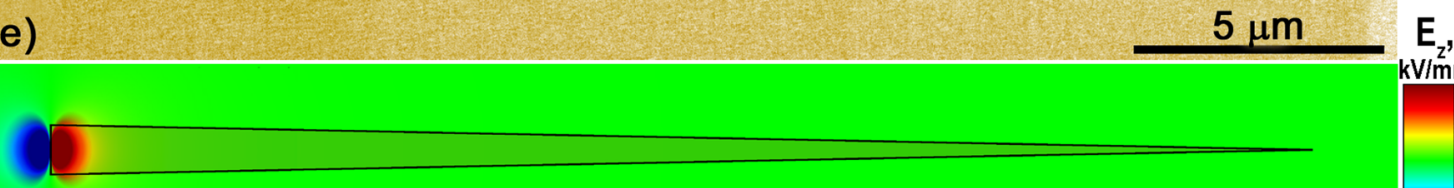

(f)

FIG. 3. Dependences of domain (a) length and (c) width on the switching pulse amplitude for a duration of $0.5 \mathrm{~s}$. Dependences of domain (b) length and (d) width on pulse duration for a pulse amplitude of $100 \mathrm{~V}$. A long wedge-like domain: (e) PFM image and (f) comparison of the spatial distribution of the polar component of the electric field produced by a biased SPM tip and the domain image. $U_{p}=200 \mathrm{~V}, t_{p}=5 \mathrm{~s} .400-\mu \mathrm{m}$ thick MgO:LN X-cut.

Such an approach was applied successfully for explanation of the domain structure evolution and an experimentally observed variety of domain shapes. ${ }^{14,45,46}$ The domain wall moves as a result of step generation and kink motion. ${ }^{4}$ For switching in the bulk, the generation of the elementary steps with one-unit-cell thickness by 2D-nucleation leads to appearance of two charged kinks: positively charged head-to-head (h2h) and negatively charged tail-to-tail ( $\mathrm{t} 2 \mathrm{t})$ [Fig. 4(a)]. The CDW tilted from the polar direction consists of regularly distributed charged kinks of the same type [Fig. 4(b)]. The kink motion is a result of $1 \mathrm{D}$-nucleation. ${ }^{4}$
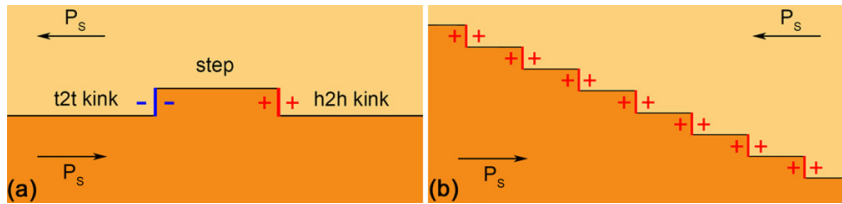

FIG. 4. (a) A moving step on the domain wall with $h 2 h$ and $t 2 t$ charged kinks and (b) a flat domain wall tilted from the main crystallographic direction with $\mathrm{h} 2 \mathrm{~h}$ kinks only. 
Both nucleation probabilities are governed by the excess of the polar component of the spatially inhomogeneous and time dependent local field averaged by the nucleus volume $E_{l o c . z}(r, t)$ over the threshold values $E_{\text {th.i }}\left(E_{\text {th.st }}\right.$ and $\left.E_{\text {th.k }}\right),{ }^{50}$

$$
\Delta E_{l o c . z . i}(r, t)=E_{l o c . z}(r, t)-E_{t h . i} .
$$

$E_{\text {loc.z }}$ consists of several inputs: (1) the external field $\left(E_{\text {ex.z }}\right)$ produced by the biased tip, (2) the depolarization field $\left(E_{\text {dep.z }}\right)$ produced by bound charges localized at the base of the wedge-like domain and charged kinks, (3) the external screening field $\left(E_{s c r . z}\right)$ governed by the fast charge redistribution on the surface near the tip by switching current and charge injection, and (4) the bulk screening field $\left(E_{b . z}\right)$ produced by a slow redistribution of the bulk screening charges and surface conductivity, ${ }^{4,14,45,46}$

$$
E_{l o c . z}(r, t)=E_{\text {ex.z }}(r, t)-\left[E_{\text {dep.z }}(r, t)-E_{\text {scr.z }}(r, t)\right]-E_{b . z}(r, t) .
$$

The domain widening by growth of the domain base is caused by the step generation stimulated by the polar component of the field produced by the biased tip. ${ }^{42}$ The field dependence of the domain widening (increase in the domain base) velocity by step generation is as follows:

$$
v_{b}(E)=\mu_{b}\left(E_{l o c . z}-E_{t h . s t}\right),
$$

where $\mu_{b}$ is the base growth mobility and $E_{t h . s t}$ is the threshold field for step generation.

The kink motion is stimulated by the depolarization field produced by charged kinks. Thus, the field dependence of the kink motion velocity is expressed by

$$
v_{k}(E)=\mu_{k}\left(E_{l o c . z}-E_{t h . k}\right)
$$

where $\mu_{k}$ is the kink motion mobility and $E_{t h . k}$ is the threshold field for kink motion.

\section{B. Computer simulation}

To define quasistationary electric field $\boldsymbol{E}$, the corresponding potential $\varphi$ can be introduced as $\boldsymbol{E}=-\nabla \varphi$. Maxwell equation: $d i v$ $\boldsymbol{D}=\rho$, where $D_{x, y}=\varepsilon_{o} \varepsilon_{11} E_{x, y}$ and $D_{z}=\varepsilon_{o} \varepsilon_{33} E_{z}$. Here, $\varepsilon_{o}=8.85 \cdot 10^{12} \mathrm{~F} / \mathrm{m}$ is the universal dielectric constant. The CLN dielectric permittivity was $\varepsilon_{11}=85$ and $\varepsilon_{33}=29$. $^{51}$ The surface charge density at the charged domain walls depends on the wall orientation: $\rho=-2 P_{s} \sin (\alpha)$, where $\alpha$ is the wall tilt from the polar direction. The boundary condition at the SPM tip surface $V_{\text {tip surf }}=U$. The grounded bottom electrode was considered by the zero potential boundary condition, $\varphi_{\mathrm{f}}(\mathrm{x}, \mathrm{y}=\mathrm{L}, \mathrm{z})=0$, where $\mathrm{L}$ is the sample thickness. The similar field simulation is presented in Ref. 24 .

The spatial distribution of $E_{l o c . z}$ for the wedge-like domain during switching by a conductive SPM tip was simulated by the finite element method using the commercial software COMSOL Multiphysics. The considered SPM tip had a conical shape with a semispherical end. The following tip parameters were used: height of the conical part of $1 \mu \mathrm{m}$, the cone angle of $40^{\circ}$, and the radius of $30 \mathrm{~nm}$. The bias voltage $U_{s w}=+100 \mathrm{~V}$. We considered the wedgelike domain with a neutral base and two charged side domain walls [Fig. 5(e)]. The domain width $(w)$ was $250 \mathrm{~nm}$, the depth at the base $(d)$ was $20 \mathrm{~nm}$, and the length $(l)$ was $3 \mu \mathrm{m}$. The depolarization field at the base was completely compensated by the external screening.

The spatial distributions of the polar component of the electric field on the Y-cut were simulated: (1) $E_{\text {ex. }}$ created by a biased tip [Fig. 5(a)], (2) $E_{\text {dep.z }}-E_{\text {scr. } z}$ created by a CDW [Fig. 5(b)], and (3) the total $E_{\text {loc.z }}$ value [Fig. 5(c)]. The spatial distribution of $E_{\text {loc.z }}$ on the domain wall is presented in Fig. 5(d).

For the forward domain growth, the domain wall motion is achieved through generation of the elementary steps on the polar
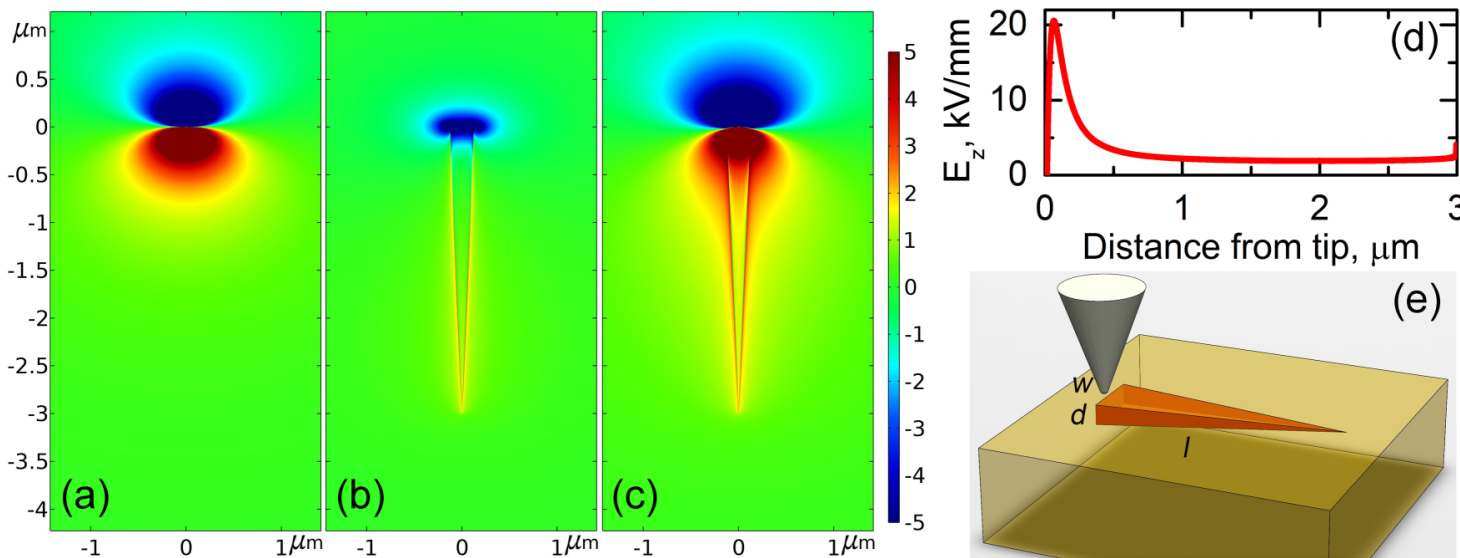

FIG. 5. The simulated spatial distributions of the polar component of the electric field on the Y-cut for a wedge-like domain during the tip-induced switching: (a) $E_{\text {ex.z }}$ created by a biased tip, (b) $E_{d e p . z}-E_{s c r . z}$ created by a CDW, and (c) total $E_{\text {loc.z }}$ value. (d) The spatial distribution of $E_{\text {loc.z }}$ on the domain wall. (e) Schematic image of the simulated model. 


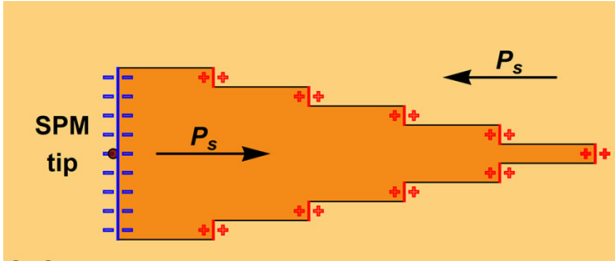

(a)

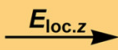

(b)
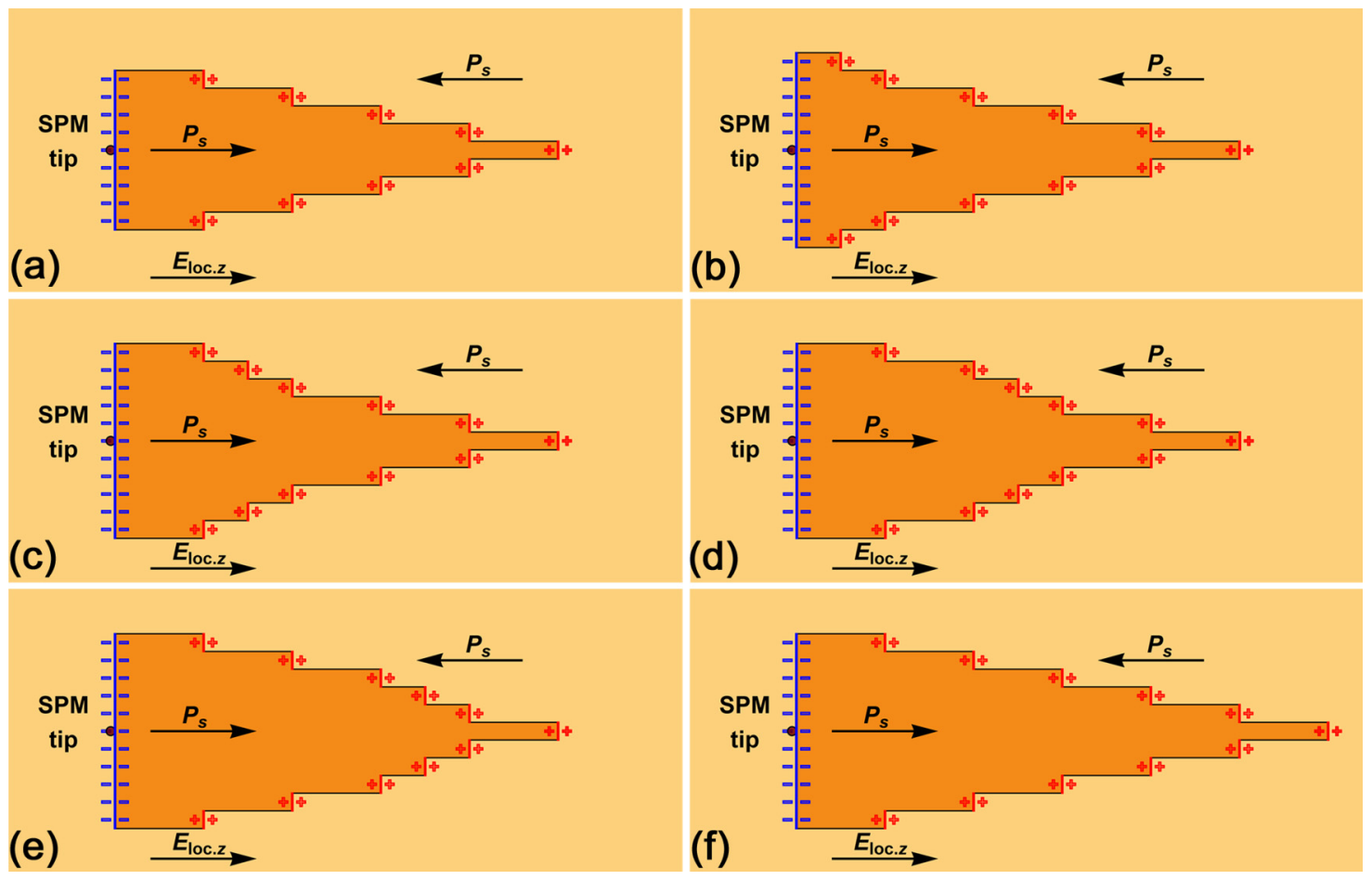

FIG. 6. A scheme of the domain forward growth on the non-polar cut of LN by step generation in the vicinity of the biased tip and motion of h2h charged kinks for a positive voltage applied: (a) the initial state, (b) generation of new steps, (c)-(e) kink motion, and (f) the final state.

surface for the polar cut and in the vicinity of the biased tip for the non-polar cut and motion of the charged kinks (Fig. 6). The elementary steps are generated at the domain walls in the vicinity of the tip [Fig. 6(b)]. The kinks move along the domain wall in the polar direction [Figs. 6(c)-6(e)].

Thus, the domain forward growth due to step generation in the field produced by the biased tip and kink motion caused by the depolarization field induced by the charged kinks allows explaining the domain growth far from the tip with a negligible value of the applied field.

The proposed mechanism can be applied also for the tip-induced switching on the polar cut of the bulk samples. In this case, for forward domain growth, it is enough to generate the steps at the domain wall on the polar surface, whereas the domain elongates by kink motion under the field produced by the charged kinks.

\section{Arrays of wedge-like domains}

The switching by scanning with a biased SPM tip leads to formation of the arrays of wedge-like domains with the length alternation (Fig. 7). There was no significant difference in the domain shape, length, and period for switching on $\mathrm{X}$ - and Y-cuts.

A linear dependence of the average length and period of the domains in arrays on the switching pulse amplitude was found [Figs. 8(a) and 8(b)]. Similar dependence of the domain length is typical for isolated domains created by the SPM tip on the LN nonpolar cuts. $^{20}$

Statistical analysis of the domain patterns [Fig. 8(c)] allowed us to reveal that the wedge-like domains in a given array can be separated by length in three groups: (1) short (S), (2) medium (M), and (3) long (L) [Fig. 8(d)]. Moreover, the periodical sequence of domains from different groups was obtained (Fig. 9). Under the used experimental conditions, several modes of the domain length alternation with various periodicities coexisted in each array: doubling, quadrupling, and chaotic [Figs. 9(a)-9(c)].

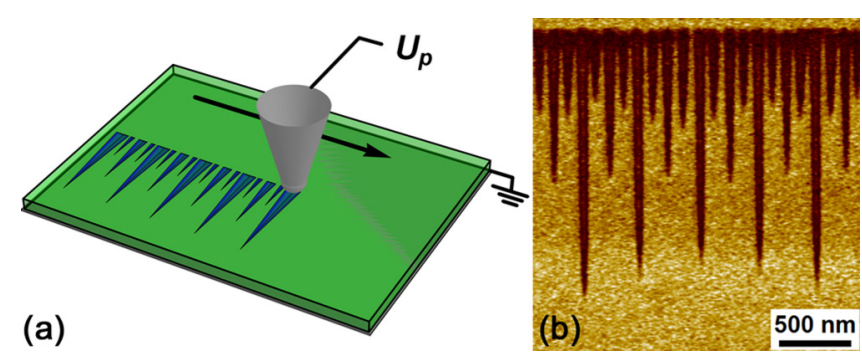

FIG. 7. (a) A scheme of the switching by scanning with a biased SPM tip and (b) a PFM image of the typical array of wedge-like domains with the length alternation. 

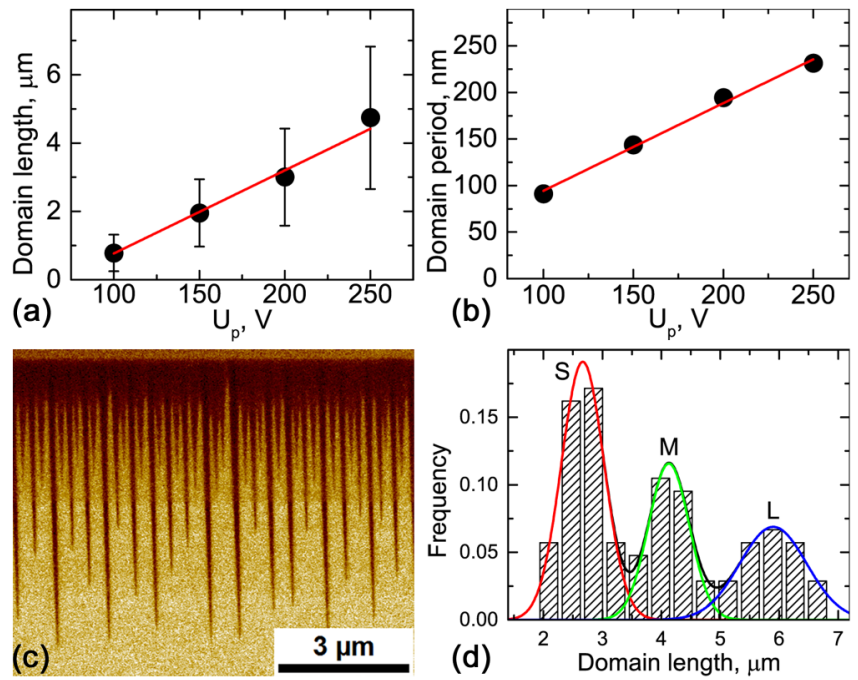

FIG. 8. The voltage dependence of (a) the average length and (b) the average period of the domains in arrays. A self-organized domain array for $U_{p}=250 \mathrm{~V}$ : (c) PFM image and (d) histogram of the domain length distribution.

The period doubling mode represents the alternation of two domain lengths [Fig. 9(a)]. The period quadrupling mode corresponds to the sequence of three domain lengths ordered in groups of four domains: L-S-M-S [Fig. 9(b)]. In the chaotic mode, the lengths of the neighboring domains were distributed irregularly [Fig. 9(c)]. The similar domain alternation mode was obtained by us earlier during scanning by a grounded SPM tip. ${ }^{28}$ This effect was attributed to an electrostatic interaction between the neighboring wedge-like domains.

The statistical analysis of the domain length in arrays allowed revealing the linear dependence of the average domain length in each group with a bias voltage [Fig. 10(a)]. The fractions of different modes in each array depend on the applied voltage. The

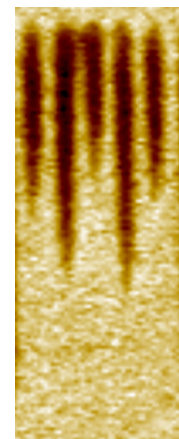

(a) $200 \mathrm{~nm}$ (b)
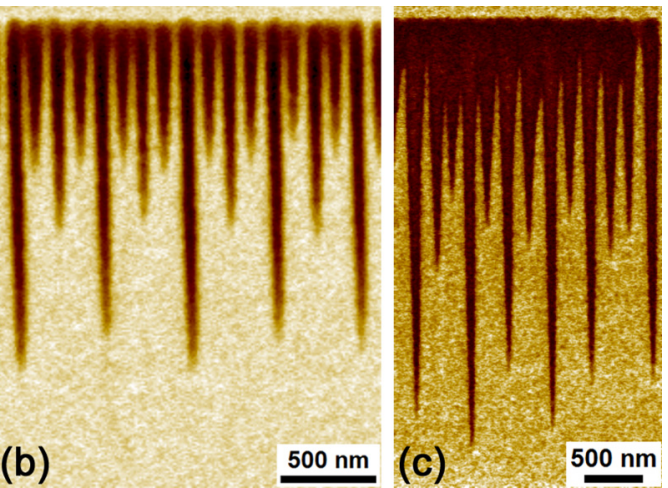

FIG. 9. PFM images of the various modes of length alternation in domain arrays: (a) doubling, (b) quadrupling, and (3) chaotic.
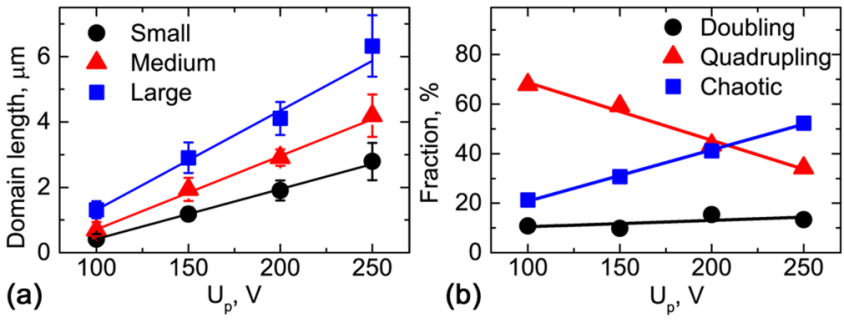

FIG. 10. The dependence of (a) the average domain length in different groups and (b) fractions of different modes in the array on the switching pulse amplitude $U_{p}$.

increasing voltage leads to a linear decrease in the quadrupling mode fraction and a linear increase in the chaotic mode fraction, while the fraction of the doubling mode remains almost constant [Fig. 10(b)].

For statistical characterization of the domain arrays, we plotted the return map (Poincaré plot) representing the recurrence plot used to reveal the self-similarity in processes. ${ }^{52}$ We plotted the dependence of the length of each domain in the given array $\left(l_{n+1}\right)$ on the length of the previous one $\left(l_{n}\right)$. The lengths were normalized on the length of the longest domain in the array (Fig. 11).

For a quadrupling mode, the alternation of the domain length in the array leads to formation of four separate clusters at the return map corresponding to four realized pairs of neighboring domains (L-S, S-M, M-S, S-L) [Fig. 11(a)]. The obtained coincidence of the cluster position at the plot with the ideal regular structure [Fig. 11(b)] proves the high ordering of the studied domain arrays.

The obtained formation of the self-assembled domain arrays can be considered in terms of the kinetic approach discussed above. In the domain array, the field at the wall of the growing domain contains the input of the depolarization fields produced by several neighboring domains. The depolarization field produced by the domain was assumed to be generated by CDW, while the domain base was completely screened. The simulation
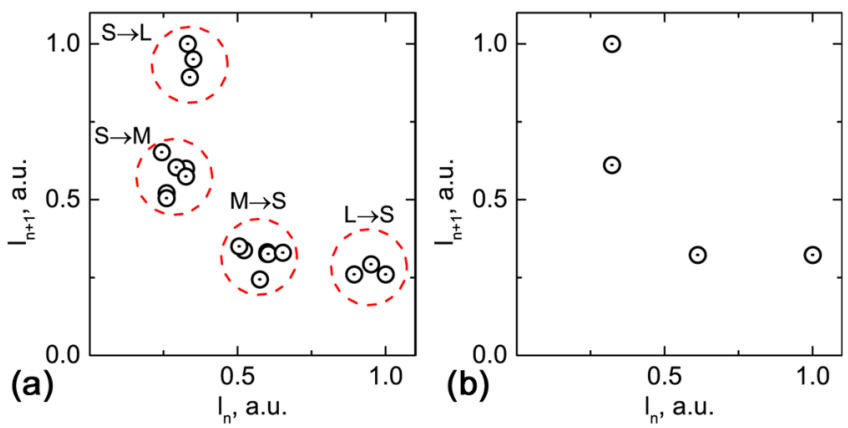

FIG. 11. Return maps for a quadruple mode: (a) for experimental domain arrays and (b) for an ideal regular array. 
demonstrates that for explanation of the observed ordering, it is necessary to consider the input of the three previous domains in the arising array ${ }^{28}$ [Fig. 6(b)]. The simulated values of $E_{l o c . z}$ for all experimentally observed combinations of three neighboring domains (L-S-M, S-M-S, M-S-L, and S-L-S) were used for calculation of the length of the appeared domain.

\section{CONCLUSION}

The forward domain growth was studied experimentally for isolated domains and domain arrays appearing as a result of tip-induced switching on the non-polar cuts of LN crystals. The domains were created and imaged with a high spatial resolution by means of scanning probe microscopy. The wedge-like domains with a high ratio of length to width (up to 30) and charged domain walls grew in a polar direction. The field produced by the biased tip with a value above the threshold for domain growth was localized in the area of about $1 \mu \mathrm{m}$ in radius, whereas the domain length was up to tens of microns. This fact was considered in terms of the kinetic approach based on analogy with crystal growth controlled by the appearance of nucleus of different dimensionalities. The wall motion by step generation and propagation of the charged kinks was discussed. The step generation rate and the kink motion velocity depend on the excess of the polar component of the local field over the threshold values. It was proposed that the local field consisted of several inputs: the external field produced by a biased tip, the depolarization field produced by charged kinks, and the screening fields. According to the simulation of the field spatial distribution, the domain forward growth is caused by the step generation in the vicinity of the tip and the kink propagation in the depolarization field produced by the neighboring kinks. This mechanism allows explaining the domain growth far from the tip with a negligible value of the external field.

It was shown that the switching by scanning with a biased SPM tip led to formation of the self-assembled arrays of the wedgelike domains with the length alternation. Three modes of the domain length alternation with various periodicities coexisted in each array: doubling, quadrupling, and chaotic. The fractions of the different modes in each array depended on the applied voltage. The statistical characterization of the domain arrays by plotting of the return map proved the high order of the studied domain arrays. The obtained formation of the self-assembled domain arrays was considered in terms of the kinetic approach taking into account the influence of the depolarization fields produced by three neighboring domains.

The proposed mechanism can be applied for the tip-induced switching on the polar cut of bulk samples. In this case, for forward domain growth, it is enough to generate the steps at the domain wall on the polar surface, whereas the domain elongates by kink motion in the field produced by the charged kinks. The obtained knowledge will allow improving the domain wall engineering methods in ferroelectric crystals.

\section{ACKNOWLEDGMENTS}

The equipment of the Ural Center for Shared Use "Modern nanotechnology" Ural Federal University was used. The research was made possible by the Russian Science Foundation (Project No. 19-12-00210).

\section{DATA AVAILABILITY}

The data that support the findings of this study are available from the corresponding author upon reasonable request.

\section{REFERENCES}

${ }^{1}$ A. K. Tagantsev, L. E. Cross, and J. Fousek, Domains in Ferroic Crystals and Thin Films (Springer, Berlin, 2010).

${ }^{2}$ R. E. Newnham, C. S. Miller, L. E. Cross, and T. W. Cline, Phys. Status Solidi 32, 69 (1975).

${ }^{3}$ S. Wada, Ferroelectrics 389, 3 (2009).

${ }^{4}$ V. Y. Shur, Advanced Piezoelectric Materials, 2nd ed. (Woodhead Publishing, 2017), pp. 235-270.

${ }^{\mathbf{5}}$ M. M. Fejer, G. A. Magel, D. H. Jundt, and R. L. Byer, IEEE J. Quantum Electron. 28, 2631 (1992).

${ }^{6}$ D. S. Hum and M. M. Fejer, C. R. Phys. 8, 180 (2007).

${ }^{7}$ V. Y. Shur, E. L. Rumyantsev, E. V. Nikolaeva, E. I. Shishkin, R. G. Batchko, G. D. Miller, M. M. Fejer, and R. L. Byer, Ferroelectrics 236, 129 (2000).

${ }^{8}$ V. Y. Shur, A. R. Akhmatkhanov, and I. S. Baturin, Appl. Phys. Rev. 2, 040604 (2015).

${ }^{9}$ M. V. Klassen-Neklyudova, M. A. Chernysheva, and A. A. Shternberg, Dokl. Akad. Nauk. SSSR 18, 527 (1948).

${ }^{10}$ B. Matthias and A. von Hippel, Phys. Rev. 73, 1378 (1948).

${ }^{11}$ W. J. Merz, Phys. Rev. 95, 690 (1954).

${ }^{12}$ E. A. Little, Phys. Rev. 98, 978 (1955).

${ }^{13}$ R. le Bihan, Ferroelectrics 97, 19 (1988).

${ }^{14}$ A. Gruverman, O. Auciello, and H. Tokumoto, Annu. Rev. Mater. Sci. 28, 101 (1998).

${ }^{15}$ A. Kholkin, S. Kalinin, A. Roelofs, and A. Gruverman, Scanning Probe Microscopy: Electrical and Electromechanical Phenomena at the Nanoscale (Springer Science \& Business Media, New York, 2007), pp. 173-214.

${ }^{16}$ P. Gao, J. Britson, J. R. Jokisaari, C. T. Nelson, S.-H. Baek, Y. Wang, C.-B. Eom, L.-Q. Chen, and X. Pan, Nat. Commun. 4, 2791 (2013).

${ }^{17}$ V. Y. Shur, Advanced Dielectric, Piezoelectric and Ferroelectric MaterialsSynthesis, Characterization \& Applications (Woodhead Publishing Ltd., Cambridge, 2008), pp. 622-669.

${ }^{18}$ V. Gopalan and T. E. Mitchell, J. Appl. Phys. 83, 941 (1998).

${ }^{19}$ V. Y. Shur, A. I. Lobov, A. G. Shur, S. Kurimura, Y. Nomura, K. Terabe, X. Y. Liu, and K. Kitamura, Appl. Phys. Lett. 87, 022905 (2005).

${ }^{20}$ D. O. Alikin, A. V. Ievlev, A. P. Turygin, A. I. Lobov, S. V. Kalinin, and V. Y. Shur, Appl. Phys. Lett. 106, 182902 (2015).

${ }^{21}$ V. G. Zalessky and S. O. Fregatov, Physica B 371, 158 (2006).

${ }^{22}$ L. S. Kokhanchik, M. V. Borodin, S. M. Shandarov, N. I. Burimov, V. V. Shcherbina, and T. R. Volk, Phys. Solid State 52, 1722 (2010).

${ }^{23}$ T. R. Volk, L. S. Kokhanchik, R. V. Gainutdinov, Y. V. Bodnarchuk, and F. Chen, Ferroelectrics 500, 129 (2016).

${ }^{24}$ A. V. Ievlev, D. O. Alikin, A. N. Morozovska, O. V. Varenyk, E. A. Eliseev, A. L. Kholkin, V. Y. Shur, and S. V. Kalinin, ACS Nano 9, 769 (2015).

${ }^{\mathbf{2 5}}$ A. P. Turygin, D. O. Alikin, Y. M. Alikin, and V. Y. Shur, Materials 10, 1143 (2017).

${ }^{26}$ M. Lilienblum and E. Soergel, J. Appl. Phys. 110, 052018 (2011).

${ }^{27}$ S. Bühlmann, E. Colla, and P. Muralt, Phys. Rev. B 72, 214120 (2005).

${ }^{28}$ A. P. Turygin, D. O. Alikin, M. S. Kosobokov, A. V. Ievlev, and V. Y. Shur, ACS Appl. Mater. Interfaces 10, 36211 (2018).

${ }^{29}$ A. V. Ievlev, A. N. Morozovska, E. A. Eliseev, V. Y. Shur, and S. V. Kalinin, Nat. Commun. 5, 4545 (2014).

${ }^{30}$ Y. Kim, S. Bühlmann, S. Hong, S. H. Kim, and K. No, Appl. Phys. Lett. 90, 072910 (2007).

${ }^{31}$ M. Abplanalp, J. Fousek, and P. Günter, Phys. Rev. Lett. 86, 5799 (2001). 
${ }^{32}$ A. V. Ievlev, A. N. Morozovska, V. Y. Shur, and S. V. Kalinin, Phys. Rev. B 91 , 214109 (2015).

${ }^{33}$ V. Y. Shur, E. L. Rumyantsev, E. V. Nikolaeva, E. I. Shishkin, D. V. Fursov, R. G. Batchko, L. A. Eyres, M. M. Fejer, and R. L. Byer, Appl. Phys. Lett. 76, 143 (2000).

${ }^{34}$ V. Y. Shur, E. L. Rumyantsev, R. G. Batchko, G. D. Miller, M. M. Fejer, and R. L. Byer, Phys. Solid State 41, 1681 (1999).

${ }^{35}$ M. Müller, E. Soergel, and K. Buse, Opt. Lett. 28, 2515 (2003).

${ }^{36}$ M. Molotskii, A. Agronin, P. Urenski, M. Shvebelman, G. Rosenman, and Y. Rosenwaks, Phys. Rev. Lett. 90, 107601 (2003).

${ }^{37}$ M. Molotskii, Y. Rosenwaks, and G. Rosenman, Annu. Rev. Mater. Res. 37, 271 (2007).

${ }^{38}$ V. Y. Shur, E. L. Rumyantsev, E. V. Nikolaeva, and E. I. Shishkin, Appl. Phys. Lett. 77, 3636 (2000).

${ }^{39}$ T. Sluka, A. K. Tagantsev, P. Bednyakov, and N. Setter, Nat. Commun. 4, 1808 (2013).

${ }^{40}$ M. P. Campbell, J. P. V. McConville, R. G. P. McQuaid, D. Prabhakaran, A. Kumar, and J. M. Gregg, Nat. Commun. 7, 13764 (2016).

${ }^{41}$ A. A. Esin, A. R. Akhmatkhanov, and V. Y. Shur, Appl. Phys. Lett. 114, 092901 (2019).
${ }^{42}$ N. A. Pertsev and A. L. Kholkin, Phys. Rev. B 88, 174109 (2013).

${ }^{43}$ A. Agronin, M. Molotskii, Y. Rosenwaks, G. Rosenman, B. J. Rodriguez, A. I. Kingon, and A. Gruverman, J. Appl. Phys. 99, 104102 (2006).

${ }^{44} \mathrm{~V}$. Y. Shur, A. V. Ievlev, E. V. Nikolaeva, E. I. Shishkin, and M. M. Neradovskiy, J. Appl. Phys. 110, 052017 (2011).

${ }^{45}$ V. Y. Shur, Nucleation Theory and Applications (Wiley-VCH, Weinheim, 2005), pp. 178-214.

${ }^{46}$ V. Y. Shur, J. Mater. Sci. 41, 199 (2006).

${ }^{47}$ E. Fatuzzo and W. J. Merz, Ferroelectricity (North-Holland Publishing Company, Amsterdam, 1967).

${ }^{48}$ R. C. Miller and G. Weinreich, Phys. Rev. 117, 1460 (1960).

${ }^{49}$ J. W. Cahn, Acta Metall. 8, 554 (1960).

${ }^{50}$ V. Y. Shur, Ferroelectric Thin Films: Synthesis and Basic Properties, Ferroelectricity and Related Phenomena (Gordon \& Breach Science Publishers, Amsterdam, 1996), Vol. 10, pp. 153-192.

${ }^{51}$ I. Tomeno and S. Matsumura, Properties of Lithium Niobate (INSPEC, The Institution of Electrical Engineers, London, 2002), pp. 129-130.

${ }^{52}$ N. Marwan, M. C. Romano, M. Thiel, and J. Kurths, Phys. Rep. 438, 237 (2007). 